\section{Large Nonlinear Optical Response in Carbon Nitride Nanotube}

Rui Hua Xie

Max-Planck-Institut für Strömungsforschung, D-37073 Göttingen

Reprint requests to Dr. R. H. X.; Fax: +49 551 5176712; E-mail: rxie@gwdg.de

Z. Naturforsch. 54a, 348-350 (1999); received March 19, 1999

The third-order optical nonlinearity of a nitrogen doped carbon nanotube is studied. It is found that carbon nitride nanotubes are potentially important in photonics owing to their large nonlinear optical response.

Key words: Fullerene; Carbon Nanotube; Hyperpolarizability; Doping Effect.

Molecules with large third-order optical nonlinearities, characterized by large second-order hyperpolarizabilities $(\gamma)$, are required for photonic applications [1]. However, the $\gamma$ magnitudes of most third-order materials are usually smaller than those needed for photonic devices. Hence, finding third-order materials with large $\gamma$ magnitudes has been a hot topic in physics and chemistry. Theoretical and experimental studies have shown that conjugated $\pi$-electron organic systems, fullerenes and carbon nanotubes, are potentially important in photonics owing to their large nonlinear optical (NLO) response [1-5].

Theoretical studies $[6,7]$ demonstrated that the lattice and electronic structures of fullerenes change with substituted doping; the band gaps and electronic polarization of the substituted fullerenes vary greatly with different substituted doping; the distribution of $\pi$ electrons on the surface of the fullerene is changed due to the substituted doping effect, the originally delocalized $\pi$ electrons in a pure fullerene becoming more localized around the substituted atoms. Obviously, these factors have a large effect on the NLO properties of fullerene-related nanotubes [5]. Recently, nitrogen or boron doped carbon nanotubes have successfully been synthesized [8]. Therefore it is interesting and useful to investigate theoretically the NLO properties of doped carbon nanotubes. In this paper we perform a calculation of the second-order hyperpolarizabilities of a single substituted zigzag nanotube (SSZN) doped by nitrogen.

The previous model [2-4] has successfully been used to describe a small zigzag nanotube: $\mathrm{C}_{60+i \times 18}$, where $i$ is a positive integer. Here the model is modified to include the effect of the dopant ions in order to describe the small SSZN, $\mathrm{C}_{59+i \times 18} \mathrm{X}$, i.e.,

$$
\begin{aligned}
H=H_{\mathrm{c}-\mathrm{c}}^{(0)}+ & H_{\mathrm{X}-\mathrm{c}}^{(1)}, \\
H_{\mathrm{c}-\mathrm{c}}^{(0)}= & \sum_{\langle i, j\rangle, s}\left(-t_{0}-\alpha_{0} y_{i j}\right)\left(c_{i, s}^{\dagger} c_{j, s}+\text { h.c. }\right) \\
& +\frac{K_{0}}{2} \sum_{\langle i, j\rangle} y_{i, j}^{2}+U_{0} \sum_{i} c_{i, \uparrow}^{\dagger} c_{i, \uparrow} c_{i, \downarrow}^{\dagger} c_{i, \downarrow} \\
& +V_{0} \sum_{\langle i j\rangle, s, s^{\prime}} c_{i, s}^{\dagger} c_{i, s} c_{j, s^{\prime}}^{\dagger} c_{j, s^{\prime}}, \\
H_{\mathrm{X}-\mathrm{c}}^{(1)}= & \sum_{\langle i j\rangle_{, s}}\left(-t_{1}-\alpha_{1} y_{i j}\right)\left(c_{i, s}^{\dagger} c_{j, s}+\text { h.c. }\right) \\
& +\frac{K_{1}}{2} \sum_{\langle i, j\rangle} y_{i, j}^{2}+U_{1} \sum_{i} c_{i, \uparrow}^{\dagger} c_{i, \uparrow} c_{i, \downarrow}^{\dagger} c_{i, \downarrow} \\
& +V_{1} \sum_{\langle i j\rangle, s, s^{\prime}} c_{i, s}^{\dagger} c_{i, s} c_{j, s^{\prime}}^{\dagger} c_{j, s^{\prime}},
\end{aligned}
$$

where the sum $\langle i j\rangle$ is taken over the nearest neighbors for both the $\mathrm{c}-\mathrm{c}$ and $\mathrm{X}-\mathrm{c}$ bonds; $t_{0}$ and $t_{1}$ represent the hopping integrals for the $\mathrm{c}-\mathrm{c}$ and $\mathrm{X}-\mathrm{c}$ bonds, respectively; $\alpha_{0}$ and $\alpha_{1}$ are the electron-phonon coupling constants related to the $\mathrm{c}-\mathrm{c}$ and $\mathrm{X}-\mathrm{c}$ bonds, respectively; $K_{0}$ and $K_{1}$ are the spring constants corresponding to the $\mathrm{c}-\mathrm{c}$ and $\mathrm{X}-\mathrm{c}$ bond, respectively; $y_{i j}$ is the change of the bond length between the $i$ th and $j$ th atom; the operator $c_{i, s}\left(c_{i, s}^{\dagger}\right)$ annihilates (creates) a $\pi$ electron at the $i$ th atom with spin $s(s=\uparrow, \downarrow) ; U_{0}$ or $U_{1}$ is the usual on-site Coulomb repulsion strength; $V_{0}$ or $V_{1}$ is the Coulomb interaction between the nearest and next-nearest atoms. Since there is only one substituted atom (i.e., $X$ ) in the zigzag nanotube, $H_{\mathrm{X}-\mathrm{c}}^{(1)}$ plays a perturbational role, and as an approximation the original empirical parameters $\left(t_{0}, \alpha_{0}, K_{0}, U_{0}\right.$, $\left.V_{0}\right)$ in $H_{\mathrm{c}-\mathrm{c}}^{(0)}$ are assumed not to change due to the substituted doping (taken to be the same in this numerical calculation as those [4] in the pure zigzag nanotube), $U_{1} \approx U_{0}$, and $V_{1} \approx V_{0}$. The choice of the three parameters $\left(t_{1}, \alpha_{1}, K_{1}\right)$ for the $\mathrm{X}-\mathrm{c}$ bonds is of course important. Using the self-consistent-field molecular-orbital (SCFMO) method, Kurita et al. [8] have calculated the molecular structure and electronic properties for $\mathrm{C}_{59} \mathrm{X}(\mathrm{X}=\mathrm{B}, \mathrm{N})$. Also we investigated the structural and electronic properties of the same substituted doped fullerenes by carefully adjusting the values of the three parameters $t_{1}, \alpha_{1}$, and $K_{1}$, and we found that our numerical calculations can accurately reproduce the results [8] obtained from 
the SCFMO method if $t_{1}=1.17 \mathrm{eV}, \alpha_{1}=6.04 \mathrm{eV} / \AA$, and $K_{1}=51.1 \mathrm{eV} / \AA^{2}$ for $\mathrm{C}_{59} \mathrm{~B}$, and $t_{1}=1.05 \mathrm{eV}$, $\alpha_{1}=6.13 \mathrm{eV} / \AA$, and $K_{1}=49.6 \mathrm{eV} / \AA^{2}$ for $\mathrm{C}_{59} \mathrm{~N}$. Therefore, in this numerical calculation, we adopt the same values of all the parameters for $\mathrm{C}_{59} \mathrm{~B}$ and $\mathrm{C}_{59} \mathrm{~N}$.

Within the independent electron approximation and sum-over-state approach, the second-order hyperpolarizability $\gamma$ can be written as $\gamma=\sum_{i=1}^{5} \gamma_{i}(-3 \omega ; \omega, \omega, \omega)$, where $\gamma_{i}$ is given in detail in [4].

Based upon the electronic structure obtained in the above model, we calculate the static second-order hyperpolarizabilities $\left(\gamma_{\text {impurity }}\right)$ of the SSZN C $59+i \times 18$ X. Here the $z$ axis is taken along the direction from the bottom hexagon to the top one, and the $x$ axis is directed to the impurity ion X. Since the ratio between the different components of $\gamma$ are not known, a spatial average of $\gamma$ is given by [2-4] $\gamma=\left[\gamma_{x x x x}+\gamma_{y y y y}+\gamma_{z z z z}+2\left(\gamma_{x x y y}+\gamma_{y y z z}+\right.\right.$ $\left.\left.\gamma_{z z x x}\right)\right] / 5$. In order to see the substituted doping effect on the NLO response of the zigzag nanotubes, we calculate the ratio $Q$ between $\gamma_{\text {impurity }}$ and $\gamma_{\text {purity }}$ where $\gamma_{\text {purity }}$ is the static $\gamma$ value of the corresponding pure nanotube, which is given by the empirical formula $\gamma_{\text {purity }}=$ $(1+i \times 18 / 60)^{2.98} \gamma_{60}$ for the zigzag nanotube [4], and $\gamma_{60}=5.6 \times 10^{-34}$ esu is the static $\gamma$ value of $\mathrm{C}_{60}$. For $i=1$, $4,8,12, Q=31.1,34.3,38.6,41.5$, respectively. It is seen that the static $\gamma$ value of the doped zigzag nanotube is more than 30 times larger than that of the corresponding pure one. This means that the substituted doping greatly increases the nonlinear optical polarizability of the carbon nanotube.

The dynamical nonlinear optical response of $\mathrm{C}_{59+5 \times 18} \mathrm{~N}$ has also been investigated by calculating the THG spectrum, and the results are shown in Figure 1. The first peak is located at $3 \omega=0.131 \mathrm{eV}$, and the corresponding $\gamma$ magnitude is $32.6 \times 10^{-32}$ esu, which is about three times larger than that $\left(=11.4 \times 10^{-32}\right.$ esu [4]) of the pure zigzag nanotube. As in the pure case, the first peak in the $\gamma$ spectrum of the SSZN is caused by a three-photon resonance between two energy levels near Fermi levels with one in the conduction band and the other in the valence band.

Moreover, it is seen that the highest peak in the $\gamma$ spectrum is located at $3 \omega=0.451 \mathrm{eV}$, and the corresponding $\gamma_{\max }$ magnitude is about $337.14 \times 10^{-30} \mathrm{esu}$, which are more than 50 times larger than that $\left(=6.66 \times 10^{-30} \mathrm{esu}\right.$ [4]) of the highest peak of the pure zigzag nanotube. This peak is caused by one-, two-, and three-photon resonance enhancement (OTTPRE) [4]. The other peaks with larg-

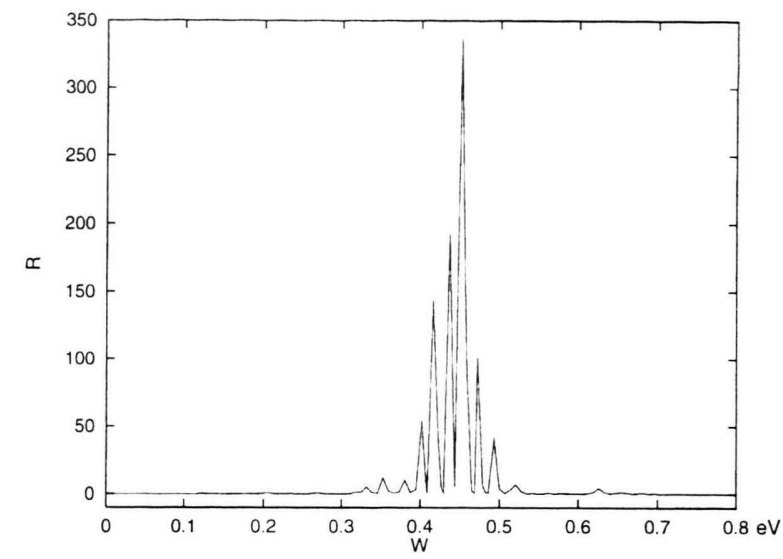

Fig. 1. The second-order hyperpolarizability $\gamma$ spectra of $\mathrm{C}_{59+5 \times 18} \mathrm{~N}$, where $R=\gamma / 10^{-30}$ esu and $W=3 \omega \mathrm{eV}$.

er $\gamma$ magnitudes are produced by two- or three-photon resonance. For example, the three-photon peaks with larger $\gamma$ magnitudes are located at $3 \omega=0.415,0.437$, $0.471 \mathrm{eV}$, and the two-photon peaks with large $\gamma$ magnitudes are located at $3 \omega=0.401,0.493 \mathrm{eV}$. Furthermore, we notice that the major response peaks with large $\gamma$ magnitudes concentrate in a narrow region, where the optical frequency is near the energy gap, i.e., $3 \omega \approx 3 E_{g}$, where $E_{g}=0.135 \mathrm{eV}$. The reason is the same as that for the pure case [4], i.e., many OTTPRE processes are observed in a doped zigzag nanotube, but only those transition processes, which occur between the energy levels near Fermi levels, are able to contribute large $\gamma$ magnitudes.

In conclusion, our numerical calculations indicate that the nitrogen doped zigzag nanotube is potentially important in photonics owing to the large nonlinear optical response. Very recently, the generation of nitrogen doped carbon nanotubes has been reported [8]. In the light of this experimental progress, the above theoretical studies are significant in the photonic application of carbon nitride nanotubes. Surely, it would be interesting to see what would happen if heavier substituted doping of carbon nanotubes were done. Based on the results in this paper, most probably this process would raise the $\gamma$ magnitude further. Work along this direction is in progress.

This work is supported by Alexander von Humboldt Foundation. 
[1] J., Messier, F. Kajzar, and P. N. Prasad, Organic Molecules for Nonlinear Optics and Photonics; Kluwer Academic, Dordrecht 1991

[2] R. H. Xie and J. Jiang, Appl. Phys. Lett. 71, 1029 (1997).

[3] R. H. Xie and Q. Rao, Appl. Phys. Lett. 72, 2358 (1998).

[4] R. H. Xie and J. Jiang, J. Appl. Phys. 83, 3001 (1998).

[5] M. Dresselhaus, G. Dresselhaus, and P. C. Eklund, Science of Fullerenes and Carbon Nanotubes; Academic Press, San Diego 1996.
[6] T. Takahashi, S. Suzuki, T. Morikawa, H. Katayama-Yoshida, S. Hasegawa, H. Inokuchi, K. Seki, K. Kikuchi, S. Suzuki, K. Ikemoto, and Y. Achiba, Phys. Rev. Lett. 68, 1232 (1992).

[7] N. Kurita, K. Kobayashi, and H. Kumahora, Chem. Phys. Lett. 198, 95 (1992).

[8] K. Suenaga, M. P. Johansson, N. Hellgren, E. Broitman, L. R. Wallenberg, C. Colliex, J. E. Sundgren, and L. Hultman, Chem. Phys. Lett. 300, 695 (1999). Related references therein. 